\title{
Zonotopic and Gaussian State Estimator: the Predictor and Filter Algorithms *
}

\author{
Alesi Augusto de Paula* Guilherme Vianna Raffo* \\ Bruno Otávio Soares Teixeira* \\ * Graduate Program in Electrical Engineering, Universidade Federal de \\ Minas Gerais \\ Av. Antônio Carlos 6627, 31270-901, Belo Horizonte, MG, Brazil \\ (e-mails: alesi@ufmg.br,raffo@ufmg.br,brunoot@ufmg.br).
}

\begin{abstract}
In the literature, the state estimator for linear systems called zonotopic and Gaussian Kalman estimator was introduced by Combastel in 2015. It was proposed as a predictor and presented in the one-step form. The difference between a predictor and a filter is the measurement sequence used to estimate states. Predictors use the past measurements, while filters use both past and current measurements. Also, in the one-step form, the states are estimated at once. In the two-step form, the states are estimated according to the forecast and data-assimilation steps, that is, the predictive/corrective structure is made explicit. This paper revisits such estimator, here called ZGKP, and proposes two novelties. First, the two-step form is introduced for the ZGKP. After, the filter version to the estimator is proposed, here called ZGKF, in the two equivalent forms. The advantages by using the filter in comparison to the predictor are discussed.
\end{abstract}

Keywords: Kalman filter; set-based estimation; linear systems; predictors; filters.

\section{INTRODUCTION}

The Kalman filter (KF) (Kalman, 1960) is the minimumvariance state estimator for linear and Gaussian systems. The KF assumes that all uncertainties are characterized by Gaussian random variables (GRV), whose support is unbounded. Then, the two first moments of the state random variable $(\mathrm{RV})$, represented by the mean and covariance matrix, define the a posteriori probability density function (PDF).

Although the KF is called filter, it was originally proposed as a predictor (Kalman, 1960). The term state estimator is employed to characterize both predictor and filter forms. The former uses only the past measurements, while the latter uses both past and current measurements. The Kalman predictor (KP) and the KF can be presented in the one-step and two-step equivalent forms (Teixeira, 2008). The first one allows recursively to compute the state estimates at once. The two-step algorithm allows to compute recursively the state estimates, making explicit the forecast and data-assimilation steps. In this case, transformations of RVs over the process and measurement models are processed individually. The process model is used to predict RVs, while the measurement model and measurement sequence are used to correct them. In order to provide current measurements for the KF, the data acquisition should be performed instantaneously. In practice, we relax it by considering that the time spent to process such measurements is insignificant in comparison to the sampling time. Thereby, the KF becomes feasible for

\footnotetext{
‡ This work has been supported by the Brazilian agencies CAPES, FAPEMIG, and CNPq.
}

most applications. However, for large-scale applications, this relaxation might be unfeasible, and thus the KP is used (Kim et al., 2008, 2009).

Another approach used to represent uncertainty is known as set membership. While Kalman's estimators provide the most likely region to estimate the states, set-based estimators provide compact sets to include guaranteedly the states. The set-membership framework assumes unknownbut-bounded (UBB) uncertainties, which are characterized by sets containing the worst-case realizations. To achieve the guaranteed estimation, such sets must be taken into account over state estimation. Usual sets in the literature are ellipsoids, strips, polyhedrons, polytopes, zonotopes, parallelotopes and intervals (Jaulin and Kieffer, 2001; Moore et al., 2009; Le et al., 2013). In this paper, we focus on zonotopes, which are sets more general than intervals and parallelotopes (Le et al., 2013). Zonotopes are closed under affine transformations and Minkowski sum, and represented with low computational burden (Kühn, 1998; Combastel, 2003). Some state estimators were proposed in (Combastel, 2003; Alamo et al., 2005; Le et al., 2012) based on zonotopes. In (de Paula et al., 2018), the KF and zonotopic estimator proposed in (Alamo et al., 2005) are revisited, in order to make arise a conceptual comparison among them.

In (Combastel, 2015a), the zonotopic Kalman estimator (ZKP) was proposed, addressed to state estimation and fault detection. In such work, the Frobenius norm is used to treat covariation matrices, which are analogous to covariance matrices. Thereby, the ZKP is seen as a minimum-variance estimator. Such algorithm was originally proposed as a predictor, though it is called filter. 
The ZKP was extended to the zonotopic and Gaussian Kalman estimator (ZGKP) in (Combastel, 2015b), being possible to consider the individual influence of both GRV and zonotopic variable $(\mathrm{ZV})$ at the same time. For this, the Kalman gain is extended to minimize both covariation and covariance matrices. Thanks to a weight parameter, the ZGKP can combine, or match, KP and ZKP.

This paper revisits the ZGKP and proposes two novelties. The first one is to introduce the two-step form for the ZGKP. After, we propose to modify its predictive structure to the filter version in the two equivalent forms. At the end, we present a numerical example to illustrate the accuracy improvement by using the filter instead of the predictor. Here, in order to differentiate predictors from filters, the ZGKF is called filter, while the ZGKP is its predictor version, which was originally proposed in (Combastel, $2015 b)$.

\section{PRELIMINARIES}

This section aims at introducing some concepts about GRV, zonotope and, finally, the combination of their realizations, called hybrid vector. In this paper, capital letters characterize RVs, while calligraphic letters characterize sets such as zonotopes. Moreover, the superscripts $h, z$, and $g$ indicate hybrid vector, ZV, and GRV, respectively.

\subsection{Random Variables}

Let $X$ be an $n$-dimensional random variable vector (RV). The mean and covariance matrix of $X$ are given by $\hat{x}=\mathrm{E}[X]$ and $P^{\mathrm{xx}}=\operatorname{cov}(X, X) \triangleq \mathrm{E}\left[(X-\hat{x})(X-\hat{x})^{\mathrm{T}}\right]$, respectively, where $\mathrm{E}[\bullet]$ is the expected value operator. As the Gaussian PDF $p(x)$ is completely characterized by the mean and covariance matrix, the GRV $X$ can be represented by $X \sim \mathcal{N}\left(\hat{x}, P^{\mathrm{xx}}\right)$.

The affine transformation and sum operations of uncorrelated GRVs are computed as

$$
\begin{aligned}
L X+m & \sim \mathcal{N}\left(L \hat{x}+m, L P^{\mathrm{xx}} L^{\mathrm{T}}\right), \\
X+W & \sim \mathcal{N}\left(\hat{x}+\hat{w}, P^{\mathrm{xx}}+P^{\mathrm{ww}}\right),
\end{aligned}
$$

where $L \in \mathbb{R}^{b \times n}, m \in \mathbb{R}^{b}$, and $W \sim \mathcal{N}\left(\hat{w}, P^{\mathrm{ww}}\right)$.

An RV with chi-square distribution for $n$ degrees of freedom is defined as

$$
X^{\chi} \triangleq(X-\hat{x})^{\mathrm{T}}\left(P^{\mathrm{xx}}\right)^{-1}(X-\hat{x})
$$

whose the realization $x^{\chi}$ satisfies $x^{\chi} \geq 0$ and $P^{\mathrm{xx}} \succ 0_{n \times n}$ is a positive-definite matrix, where $0_{n \times n}$ represents a null matrix. It can be abbreviated by $X^{\chi} \sim \chi_{n}^{2}(n, 2 n)$, where $\mathrm{E}\left[X^{\chi}\right]=n$ and $\operatorname{cov}\left(X^{\chi}, X^{\chi}\right)=2 n$.

The cumulative distribution function (CDF) $c(x)$ of a given $\mathrm{PDF} p(x)$ is defined as

$$
c(x) \triangleq \int_{-\infty}^{x_{n}} \ldots \int_{-\infty}^{x_{1}} p\left(x_{1}, \ldots, x_{n}\right) d x_{1} \ldots d x_{n}
$$

such that $0 \leq c(x) \leq 1, \forall x \in \mathbb{R}^{n}$. Due to the cumulative characteristic, all CDFs are monotonically increasing.

\subsection{Zonotopes}

An $n$-dimensional unitary box $\mathcal{B}^{n} \triangleq[-1,1]^{n}$ is defined as $n$ unitary intervals. The Minkowski sum of two sets is defined as $\mathcal{S}_{1} \oplus \mathcal{S}_{2} \triangleq\left\{s_{1}+s_{2}: s_{1} \in \mathcal{S}_{1}, s_{2} \in \mathcal{S}_{2}\right\}$. The Pontryagin difference of two sets is defined as $\mathcal{S}_{1} \ominus \mathcal{S}_{2} \triangleq\{x: x+$ $\left.s_{2} \in \mathcal{S}_{1}, \forall s_{2} \in \mathcal{S}_{2}\right\}$. Given a set $\mathcal{S} \subset \mathbb{R}^{n}$ and a matrix $L \in \mathbb{R}^{b \times n}$, the linear transformation of $\mathcal{S}$ is defined as $L \mathcal{S}=\{L s: s \in \mathcal{S}\}$.

The zonotope $\mathcal{X}$ is defined as an affine transformation of the unitary box $\mathcal{B}^{n_{g}^{\mathrm{x}}}$, that is,

$$
\mathcal{X} \triangleq\left\{\hat{x}, G^{\mathrm{x}}\right\}=\hat{x} \oplus G^{\mathrm{x}} \mathcal{B}^{n_{g}^{\mathrm{x}}},
$$

where $\hat{x} \in \mathbb{R}^{n}$ and $G^{\mathrm{x}} \in \mathbb{R}^{n \times n_{g}^{\mathrm{x}}}$ are called center and generator matrix, respectively. Furthermore, each column of $G^{\mathrm{x}}, g_{j}^{\mathrm{x}}=\operatorname{col}_{j}\left(G^{\mathrm{x}}\right)$, is called generator.

Special cases of zonotopes are intervals, for $G^{\mathrm{x}}$ diagonal, and parallelotopes, for $G^{\mathrm{x}}$ square and invertible. Moreover, the smallest interval containing the zonotope $\mathcal{X}$ is called interval hull and denoted by

$$
\triangle \mathcal{X}=\hat{x} \oplus \operatorname{rs}\left(G^{\mathrm{x}}\right) \mathcal{B}^{n}
$$

where $\operatorname{rs}\left(G^{\mathrm{x}}\right)_{i, i}=\sum_{j=1}^{n_{g}}\left|g_{i, j}^{\mathrm{x}}\right|$, and $|\bullet|$ is the absolute value operator. This procedure guarantees the inclusion $\mathcal{X} \subset \Delta \mathcal{X}$.

The affine transformation and sum operations of zonotopes are computed as

$$
\begin{aligned}
L \mathcal{X} \oplus m & =\left\{L \hat{x}+m, L G^{\mathrm{x}}\right\}, \\
\mathcal{X} \oplus \mathcal{W} & =\left\{\hat{x}+\hat{w},\left[G^{\mathrm{x}} G^{\mathrm{w}}\right]\right\},
\end{aligned}
$$

where $\mathcal{W}=\left\{\hat{w}, G^{\mathrm{w}}\right\}$

The Frobenius norm of a matrix $M \in \mathbb{R}^{a \times b},\|\bullet\|_{\mathrm{F}}$, is defined as

$$
\|M\|_{\mathrm{F}} \triangleq \sqrt{\operatorname{tr}\left(M M^{\mathrm{T}}\right)}=\sqrt{\operatorname{tr}\left(M^{\mathrm{T}} M\right)},
$$

where $\operatorname{tr}(\bullet)$ is the trace of a matrix. When $M$ is a generator matrix, the multiplication $M M^{\mathrm{T}}$ is called covariaton (Combastel, 2015a).

Given the zonotope $\mathcal{X}=\left\{\hat{x}, G^{\mathrm{x}}\right\}$, we can reduce the generator number $n_{g}$ of $G^{\mathrm{x}}$ to the desired order $\varphi_{g}$, yielding the reduced order zonotope $\overline{\mathcal{X}}=\left\{\hat{x}, \bar{G}^{\mathrm{x}}\right\}$. This procedure increases the computational speed and satisfies the inclusion property $\mathcal{X} \subset \overline{\mathcal{X}}$. The reduction algorithm is presented in (de Paula et al., 2018) and abbreviated by $\bar{G}^{\mathrm{x}}=\operatorname{red} \_z o n\left(G^{\mathrm{x}}, \varphi_{g}\right)$.

\subsection{Hybrid Vector}

In order to combine a GRV and a ZV, the hybrid vector is defined as follows.

Definition 1. (Combastel, 2015b) Consider the ZV $\mathcal{X}_{k}=$ $\left\{0_{n \times 1}, G_{k}^{\mathrm{x}}\right\}$ and the GRV $X_{k} \sim \mathcal{N}\left(0_{n \times 1}, P_{k}^{\mathrm{gg}}\right)$. Given the realizations $x_{k}^{\mathrm{z}}$ and $x_{k}^{\mathrm{g}}$, from $\mathcal{X}_{k}$ and $X_{k}$, respectively, the hybrid vector $x_{k}^{\mathrm{h}}$ is defined as

$$
x_{k}^{\mathrm{h}} \triangleq \hat{x}_{k}^{\mathrm{h}}+x_{k}^{\mathrm{z}}+x_{k}^{\mathrm{g}},
$$

where $\hat{x}_{k}^{\mathrm{h}} \in \mathbb{R}^{n}$ is the deterministic center of $x_{k}^{\mathrm{h}}$.

Note that the hybrid vector (10) is defined as a sum of realizations. To characterize it, we must split each realization in terms of deterministic, zonotopic and Gaussian parcels. As the support of GRVs is unbounded, we present the confidence ellipsoid to approximate GRVs by a bounded set. 
Definition 2. (Combastel, 2015b) Consider the GRV $X \sim$ $\mathcal{N}\left(\hat{x}, P^{\mathrm{gg}}\right)$, where $P^{\mathrm{gg}} \succ 0_{n \times n}$, and the significance level, or type I error, $\alpha \in[0,1]$. The confidence ellipsoid is defined as

$\mathcal{E} \triangleq\left\{\hat{x}, P^{\mathrm{gg}}, \alpha\right\}=\left\{x \in \mathbb{R}^{n}:(x-\hat{x})^{\mathrm{T}}\left(P^{\mathrm{gg}}\right)^{-1}(x-\hat{x}) \leq \varsigma\right\}$,

where $\varsigma \in \mathbb{R}$ is the greatest value for the chi-square $R V$ with $n$ degrees of freedom (3), taken from the CDF (4) with confidence level of $(1-\alpha)$, such that the probability $p(x \in \mathcal{E})=(1-\alpha)$ is satisfied.

Thereby, we present the confidence box, in order to merge two bounded sets.

Theorem 1. (Combastel, 2015b) Let the hybrid vector $x^{\mathrm{h}}$ and the confidence ellipsoid $\mathcal{E}$ be defined as (10) and (11), respectively. Then, the confidence box $\mathcal{I}^{\alpha}$ is given by

$$
\mathcal{I}^{\alpha}=\hat{x}^{\mathrm{h}} \oplus \triangle \mathcal{X} \oplus \triangle \mathcal{E}
$$

where $\triangle \mathcal{X}$ is the interval hull of $\mathcal{X}$ given by (6),

$$
\triangle \mathcal{E}=\left\{0_{n \times 1}, G^{\mathrm{g}}\right\}
$$

is an interval enclosure containing $\mathcal{E}, G^{\mathrm{g}}=\sqrt{\varsigma} \operatorname{diag}^{\frac{1}{2}}\left(P^{\mathrm{gg}}\right)$, $\sqrt{\varsigma}$ is the square root of $\varsigma \in \mathbb{R}$ defined in $(11)$, and $\operatorname{diag}^{\frac{1}{2}}(\bullet)$ the square root of each diagonal element of the matrix $P^{\mathrm{gg}}$.

The confidence box can be used to sketch states over time. Now, an example is proposed to illustrate the procedure to obtain the confidence box (12).

Example 1. Let the hybrid state vector (10) be characterized by $\hat{x}^{\mathrm{h}}=\left[\begin{array}{l}2 \\ 3\end{array}\right], \mathcal{X}=\left\{\left[\begin{array}{l}0 \\ 0\end{array}\right],\left[\begin{array}{ccc}1 & 0 & 1 \\ -1 & 1 & 0\end{array}\right]\right\}$, and $X \sim$ $\mathcal{N}\left(0_{2 \times 1}, \mathrm{I}_{2 \times 2}\right)$. In Figure 1 , the center $\hat{x}^{\mathrm{h}}$, and both GRV $X$ and ZV $\mathcal{X}$ are illustrated, being the GRV sketched for one standard deviation. First, the interval hull of $\mathcal{X}$ is calculated. After, an interval enclosure for $X$ is provided according to the value $\alpha \in[0,1]$; in this case $\alpha=0.0027$, that is, the usual confidence level $3 \sigma$. Finally, the two intervals are summed with the center $\hat{x}^{\mathrm{h}}$, obtaining the confidence box.

In order to lead the hybrid vector $x_{k}^{\mathrm{h}}$ to an optimization problem, the following objective function $J_{k}^{\mathrm{zg}}$ is proposed by Combastel (2015b).

Definition 3. (Combastel, 2015b) Consider the ZV $\mathcal{X}_{k}=$ $\left\{0_{n \times 1}, G_{k}^{\mathrm{x}}\right\}$, the GRV $X_{k} \sim \mathcal{N}\left(0_{n \times 1}, P_{k}^{\mathrm{gg}}\right)$, and the weight parameter $\eta \in[0,1]$. The minimum-variance objective function $J_{k}^{\mathrm{zg}}$ is defined as

$$
J_{k}^{\mathrm{zg}}\left(K_{k}\right) \triangleq(1-\eta) \operatorname{tr}\left(G_{k}^{\mathrm{x}}\left(G_{k}^{\mathrm{x}}\right)^{\mathrm{T}}\right)+\eta \operatorname{tr}\left(P_{k}^{\mathrm{gg}}\right),
$$

where $K_{k}$ is the gain that minimizes $J_{k}^{\mathrm{zg}}$.

The parameter $\eta$ is chosen by the user, in order to attribute less or more weight to each uncertainty. If $\eta=1$, then, we assume no influence from ZVs. On the other hand, if $\eta=0$, then, we assume no influence from GRVs.

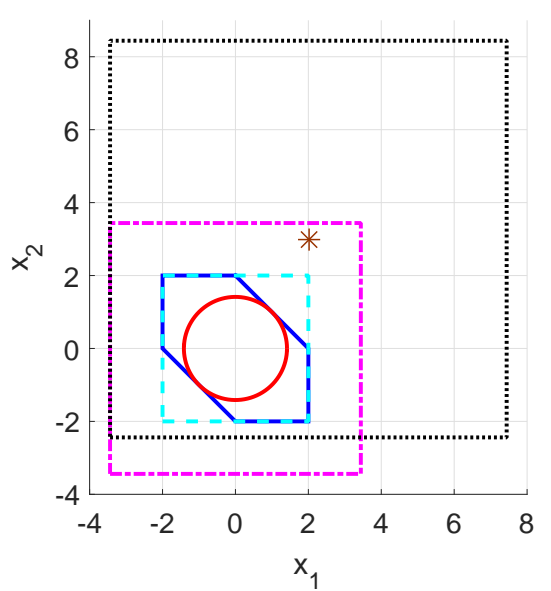

Figure 1. Illustrative example to compute the confidence box. First, the smallest box (cyan dashed line) containing $\mathcal{X}$ (blue solid line) is computed. After, an interval enclosure (magenta dash-dotted line) is provided to the GRV $X$ (red solid line). This interval is calculated according to the usual confidence level $3 \sigma$, or $\alpha=0.0027$, while the GRV is illustrated with its own standard deviation, that is, $1 \sigma$. Finally, the two computed intervals are summed up, and after shifted to the center $\hat{x}^{\mathrm{h}}(*)$, yielding the confidence box (black dotted line).

\section{PROBLEM FORMULATION}

Consider the discrete-time uncertain linear system as

$$
\begin{aligned}
& x_{k}=A_{k-1} x_{k-1}+B_{k-1} u_{k-1}+w_{k-1}^{\mathrm{z}}+w_{k-1}^{\mathrm{g}}, \\
& y_{k}=C_{k} x_{k}+v_{k}^{\mathrm{z}}+v_{k}^{\mathrm{g}},
\end{aligned}
$$

where $A_{k-1} \in \mathbb{R}^{n \times n}, B_{k-1} \in \mathbb{R}^{n \times p}$, and $C_{k} \in \mathbb{R}^{m \times n}$ are time-varying matrices, $u_{k-1} \in \mathbb{R}^{p}$ is the input vector, $y_{k} \in \mathbb{R}^{m}$ is the output vector, and $x_{k} \in \mathbb{R}^{n}$ is the state vector to be estimated. We assume known the input and output vectors, and the time-varying matrices $\forall k \geq 1$.

The variables $w_{k-1} \in \mathbb{R}^{n}$ and $v_{k} \in \mathbb{R}^{m}$ correspond to the process and measurement noise terms, respectively. We assume that there exist two types of uncertainty, namely bounded and unbounded. The former is characterized by a ZV, while the latter is characterized by a GRV.

The Gaussian noise terms $w_{k-1}^{\mathrm{g}}$ and $v_{k}^{\mathrm{g}}$ are assumed to be white and uncorrelated stochastic processes, and characterized by the GRVs $W_{k-1} \sim \mathcal{N}\left(0_{n \times 1}, Q_{k-1}\right)$ and $V_{k} \sim \mathcal{N}\left(0_{m \times 1}, R_{k}\right)$, respectively, where $Q_{k-1} \succeq 0_{n \times n}$ is a positive semi-definite matrix and $R_{k} \succ 0_{m \times m}$. On the other hand, the zonotopic noise terms $w_{k-1}^{\mathrm{z}}$ and $v_{k}^{\mathrm{z}}$ are assumed to be bounded by the zonotopes $\mathcal{W}_{k-1}=$ $\left\{0_{n \times 1}, G_{k-1}^{\mathrm{w}}\right\}$ and $\mathcal{V}_{k}=\left\{0_{m \times 1}, G_{k}^{\mathrm{v}}\right\}$, respectively, where $G_{k-1}^{\mathrm{w}}\left(G_{k-1}^{\mathrm{w}}\right)^{\mathrm{T}} \succeq 0_{n \times n}$ and $G_{k}^{\mathrm{v}}\left(G_{k}^{\mathrm{v}}\right)^{\mathrm{T}} \succ 0_{m \times m}$. In turn, the initial states $x_{0}$ are assumed to be hybrid as (10), where $P_{0}^{\mathrm{gg}} \succ 0_{n \times n}$.

In order to provide a hybrid state estimate $\forall k \geq 1$, both ZGKP and ZGKF aim at finding the Kalman gain $K_{k}$ that minimizes the objective function $J_{k}^{\mathrm{zg}}(14)$. To achieve that, each estimator is formulated according to an observer structure. From the observer point of view, the 
difference between ZGKP and ZGKF is the time step to the predictions and measurements. The ZGKP considers the measurements delayed one step $y_{k-1}$ compared to the predictions $x_{k}$, while the ZGKF considers both measurements and predictions at the same time step $k$.

For the ZGKP, the following Luenberger-based observer is proposed by Combastel (2015b):

$$
\begin{aligned}
x_{k}^{\mathrm{h}} & =A_{k-1} x_{k-1}^{\mathrm{h}}+B_{k-1} u_{k-1}+w_{k-1}^{\mathrm{z}}+w_{k-1}^{\mathrm{g}} \\
& +K_{k-1}\left(y_{k-1}-C_{k-1} x_{k-1}^{\mathrm{h}}-v_{k-1}^{\mathrm{z}}-v_{k-1}^{\mathrm{g}}\right) .
\end{aligned}
$$

For the ZGKF, the following observer is considered:

$$
\begin{aligned}
x_{k}^{\mathrm{h}} & =A_{k-1} x_{k-1}^{\mathrm{h}}+B_{k-1} u_{k-1}+w_{k-1}^{\mathrm{z}}+w_{k-1}^{\mathrm{g}} \\
& +K_{k}\left(y_{k}-C_{k} x_{k}^{\mathrm{h}}-v_{k}^{\mathrm{z}}-v_{k}^{\mathrm{g}}\right) .
\end{aligned}
$$

The main idea of observers is to correct the state prediction, given by the process model, based on measurement error. This correction is weighted by the Kalman gain. To obtain the state estimates, the unknown noise terms are replaced by their uncertainty representation.

Since $x_{0}$ is a hybrid vector, the vectors $x_{k}$ and $y_{k}$, given by (15) and (16) respectively, are hybrid too. Thus, at combining such vectors, each observer, (17) or (18), yields the hybrid state vector $x_{k}^{\mathrm{h}}$. In both cases, the GRVs $x_{0}^{\mathrm{g}}$, $w_{k-1}^{\mathrm{g}}$, and $v_{k}^{\mathrm{g}}$ are assumed to be white and uncorrelated $\forall k \geq 1$.

\section{THE ZGKP FOR DISCRETE-TIME LINEAR SYSTEMS}

From the linear estimator (17), we decompose the deterministic, zonotopic and Gaussian parcels of $x_{k}^{\mathrm{h}}$, yielding

$$
\begin{aligned}
& \hat{x}_{k}^{\mathrm{h}}=A_{k-1} \hat{x}_{k-1}^{\mathrm{h}}+B_{k-1} u_{k-1}+K_{k-1}\left(y_{k-1}-C_{k-1} \hat{x}_{k-1}^{\mathrm{h}}\right), \\
& x_{k}^{\mathrm{z}}=A_{k-1} x_{k-1}^{\mathrm{z}}+w_{k-1}^{\mathrm{z}}-K_{k-1}\left(C_{k-1} x_{k-1}^{\mathrm{z}}+v_{k-1}^{\mathrm{z}}\right), \\
& x_{k}^{\mathrm{g}}=A_{k-1} x_{k-1}^{\mathrm{g}}+w_{k-1}^{\mathrm{g}}-K_{k-1}\left(C_{k-1} x_{k-1}^{\mathrm{g}}+v_{k-1}^{\mathrm{g}}\right) .
\end{aligned}
$$

In this case, the Kalman gain $K_{k-1}$ is given by (Combastel, 2015b)

$$
K_{k-1}=A_{k-1} P_{k-1}^{\mathrm{xx}} C_{k-1}^{\mathrm{T}}\left(C_{k-1} P_{k-1}^{\mathrm{xx}} C_{k-1}^{\mathrm{T}}+R_{k-1}^{\mathrm{zg}}\right)^{-1},
$$

where

$$
\begin{aligned}
\bar{G}_{k-1}^{\mathrm{x}} & =\operatorname{red} \_ \text {zon }\left(G_{k-1}^{\mathrm{x}}, \varphi_{g}\right), \\
P_{k-1}^{\mathrm{xx}} & =(1-\eta)\left(\bar{G}_{k-1}^{\mathrm{x}}\left(\bar{G}_{k-1}^{\mathrm{x}}\right)^{\mathrm{T}}\right)+\eta P_{k-1}^{\mathrm{gg}}, \\
R_{k-1}^{\mathrm{zg}} & =(1-\eta)\left(G_{k-1}^{\mathrm{v}}\left(G_{k-1}^{\mathrm{v}}\right)^{\mathrm{T}}\right)+\eta R_{k-1} .
\end{aligned}
$$

Now, we present the equivalent forms to estimate $\hat{x}_{k}^{\mathrm{h}}, \mathcal{X}_{k}=$ $\left\{0_{n \times 1}, G_{k}^{\mathrm{x}}\right\}$, and $X_{k} \sim \mathcal{N}\left(0_{n \times 1}, P_{k}^{\mathrm{gg}}\right)$, which characterize the hybrid state vector $x_{k}^{\mathrm{h}}$. First, the two-step form is presented, since it is the most intuitive. Next, the one-step form is presented, whose predictive/corrective structure is implicit.

Algorithm 1. ZGKP: two-step form (Combastel, 2015b). This algorithm is summarized as

$$
\begin{gathered}
{\left[\hat{x}_{k}^{\mathrm{h}}, G_{k}^{\mathrm{x}}, P_{k}^{\mathrm{gg}}\right]=\text { ZGKP_TS }\left(\hat{x}_{k-1}^{\mathrm{h}}, G_{k-1}^{\mathrm{x}}, P_{k-1}^{\mathrm{gg}}, A_{k-1},\right.} \\
\left.B_{k-1}, C_{k-1}, G_{k-1}^{\mathrm{w}}, G_{k-1}^{\mathrm{v}}, Q_{k-1}, R_{k-1}, u_{k-1}, y_{k-1}, \varphi_{g}, \eta\right) .
\end{gathered}
$$

\section{Forecast step:}

1: Compute the a priori center

$$
\hat{x}_{k \mid k-1}^{\mathrm{h}}=A_{k-1} \hat{x}_{k-1}^{\mathrm{h}}+B_{k-1} u_{k-1} \text {. }
$$

2: Compute the matrix $\bar{G}_{k-1}^{\mathrm{x}}(23)$ to obtain the a priori generator matrix

$$
G_{k \mid k-1}^{\mathrm{x}}=\left[A_{k-1} \bar{G}_{k-1}^{\mathrm{x}} G_{k-1}^{\mathrm{w}}\right] .
$$

3: Compute the a priori covariance matrix

$$
P_{k \mid k-1}^{\mathrm{gg}}=A_{k-1} P_{k-1}^{\mathrm{gg}} A_{k-1}^{\mathrm{T}}+Q_{k-1} .
$$

\section{Data-assimilation step:}

1: Compute the matrices $P_{k-1}^{\mathrm{xx}}(24)$ and $R_{k-1}^{\mathrm{zg}}(25)$ to obtain the Kalman gain $K_{k-1}(22)$.

2: Compute the a posteriori estimates

$$
\begin{aligned}
\hat{x}_{k}^{\mathrm{h}} & =\hat{x}_{k \mid k-1}^{\mathrm{h}}+K_{k-1}\left(y_{k-1}-C_{k-1} \hat{x}_{k-1}^{\mathrm{h}}\right), \\
G_{k}^{\mathrm{x}} & =\left[G_{k \mid k-1}^{\mathrm{x}}-K_{k-1} C_{k-1} \bar{G}_{k-1}^{\mathrm{x}}-K_{k-1} G_{k-1}^{\mathrm{v}}\right], \\
P_{k}^{\mathrm{gg}} & =P_{k \mid k-1}^{\mathrm{gg}}-K_{k-1} C_{k-1} P_{k-1}^{\mathrm{gg}} A_{k-1}^{\mathrm{T}} .
\end{aligned}
$$

Algorithm 2. ZGKP: one-step form (Combastel, 2015b). This algorithm is summarized as

$$
\begin{gathered}
{\left[\hat{x}_{k}^{\mathrm{h}}, G_{k}^{\mathrm{x}}, P_{k}^{\mathrm{gg}}\right]=\mathrm{ZGKP} \_\mathrm{OS}\left(\hat{x}_{k-1}^{\mathrm{h}}, G_{k-1}^{\mathrm{x}}, P_{k-1}^{\mathrm{gg}}, A_{k-1},\right.} \\
\left.B_{k-1}, C_{k-1}, G_{k-1}^{\mathrm{w}}, G_{k-1}^{\mathrm{v}}, Q_{k-1}, R_{k-1}, u_{k-1}, y_{k-1}, \varphi_{g}, \eta\right) .
\end{gathered}
$$

1: Compute the matrices $\bar{G}_{k-1}^{\mathrm{x}}(23), P_{k-1}^{\mathrm{xx}}(24)$, and $R_{k-1}^{\mathrm{zg}}$ (25) to obtain the Kalman gain $K_{k-1}(22)$.

2: Compute the a posteriori estimates

$$
\begin{aligned}
\hat{x}_{k}^{\mathrm{h}} & =\left(A_{k-1}-K_{k-1} C_{k-1}\right) \hat{x}_{k-1}^{\mathrm{h}}+B_{k-1} u_{k-1}+K_{k-1} y_{k-1}, \\
G_{k}^{\mathrm{x}} & =\left[\left(A_{k-1}-K_{k-1} C_{k-1}\right) \bar{G}_{k-1}^{\mathrm{x}} G_{k-1}^{\mathrm{w}}-K_{k-1} G_{k-1}^{\mathrm{v}}\right], \\
P_{k}^{\mathrm{gg}} & =\left(A_{k-1}-K_{k-1} C_{k-1}\right) P_{k-1}^{\mathrm{gg}} A_{k-1}^{\mathrm{T}}+Q_{k-1} .
\end{aligned}
$$

\section{THE ZGKF FOR DISCRETE-TIME LINEAR SYSTEMS}

In this section, we propose to modify the ZGKP to the ZGKF. Substituting (15) at the right-hand side of (18), we made explicit the deterministic and uncertain parcels of the observer as

$$
\begin{aligned}
\hat{x}_{k}^{\mathrm{h}} & =A_{k-1} \hat{x}_{k-1}^{\mathrm{h}}+B_{k-1} u_{k-1} \\
& +K_{k}\left(y_{k}-C_{k}\left(A_{k-1} \hat{x}_{k-1}^{\mathrm{h}}+B_{k-1} u_{k-1}\right)\right), \\
x_{k}^{\mathrm{z}} & =A_{k-1} x_{k-1}^{\mathrm{z}}+w_{k-1}^{\mathrm{z}} \\
& -K_{k}\left(C_{k}\left(A_{k-1} x_{k-1}^{\mathrm{z}}+w_{k-1}^{\mathrm{z}}\right)+v_{k}^{\mathrm{z}}\right), \\
x_{k}^{\mathrm{g}} & =A_{k-1} x_{k-1}^{\mathrm{g}}+w_{k-1}^{\mathrm{g}} \\
& -K_{k}\left(C_{k}\left(A_{k-1} x_{k-1}^{\mathrm{g}}+w_{k-1}^{\mathrm{g}}\right)+v_{k}^{\mathrm{g}}\right) .
\end{aligned}
$$

By superposition, summing $\hat{x}_{k}^{\mathrm{h}}, x_{k}^{\mathrm{z}}$, and $x_{k}^{\mathrm{g}}$ results in $x_{k}^{\mathrm{h}}$. The realizations $x_{k}^{\mathrm{z}}$ and $x_{k}^{\mathrm{g}}$ are characterized by the following variables:

$$
\begin{aligned}
\mathcal{X}_{k} & =\left(\mathrm{I}_{n \times n}-K_{k} C_{k}\right)\left(A_{k-1} \mathcal{X}_{k-1} \oplus \mathcal{W}_{k-1}\right) \ominus K_{k} \mathcal{V}_{k}, \\
X_{k} & =\left(\mathrm{I}_{n \times n}-K_{k} C_{k}\right)\left(A_{k-1} X_{k-1}+W_{k-1}\right)-K_{k} V_{k} .
\end{aligned}
$$

Applying the operations (7)-(8) on $\mathcal{X}_{k}=\left\{0_{n \times 1}, G_{k}^{\mathrm{x}}\right\}$, and (1)-(2) on $X_{k} \sim \mathcal{N}\left(0_{n \times 1}, P_{k}^{\text {gg }}\right)$, we obtain the matrices 
$G_{k}^{\mathrm{x}}=\left[\left(\mathrm{I}_{n \times n}-K_{k} C_{k}\right)\left[A_{k-1} G_{k-1}^{\mathrm{x}} G_{k-1}^{\mathrm{w}}\right]-K_{k} G_{k}^{\mathrm{v}}\right]$,

$P_{k}^{\mathrm{gg}}=\left(\mathrm{I}_{n \times n}-K_{k} C_{k}\right)\left(A_{k-1} P_{k-1}^{\mathrm{gg}} A_{k-1}^{\mathrm{T}}+Q_{k-1}\right)\left(\mathrm{I}_{n \times n}-K_{k} C_{k}\right)^{\mathrm{T}}$

$$
+K_{k} R_{k} K_{k}^{\mathrm{T}} \text {. }
$$

In order to increase the computational speed, the order reduction (23) is applied on $\mathcal{X}_{k-1}=\left\{0_{n \times 1}, G_{k-1}^{\mathrm{x}}\right\}$, yielding $\overline{\mathcal{X}}_{k-1}=\left\{0_{n \times 1}, \bar{G}_{k-1}^{\mathrm{x}}\right\}$. Given $G_{k}^{\mathrm{x}}, P_{k}^{\mathrm{gg}}$, and the relation $\operatorname{tr}(M)+\operatorname{tr}(N)=\operatorname{tr}(M+N)$, the objective function $J_{k}^{\mathrm{zg}}$ (14) is written as

$$
\begin{aligned}
J_{k}^{\mathrm{zg}} & =\operatorname{tr}\left(\left(\mathrm{I}_{n \times n}-K_{k} C_{k}\right)\left(A_{k-1} P_{k-1}^{\mathrm{xx}} A_{k-1}^{\mathrm{T}}+Q_{k-1}^{\mathrm{zg}}\right)\right. \\
& \left.\times\left(\mathrm{I}_{n \times n}-K_{k} C_{k}\right)^{\mathrm{T}}+K_{k} R_{k}^{\mathrm{zg}} K_{k}^{\mathrm{T}}\right),
\end{aligned}
$$

where $P_{k-1}^{\mathrm{xx}}$ is given by (24), and

$$
\begin{aligned}
Q_{k-1}^{\mathrm{zg}} & =(1-\eta)\left(G_{k-1}^{\mathrm{w}}\left(G_{k-1}^{\mathrm{w}}\right)^{\mathrm{T}}\right)+\eta Q_{k-1}, \\
R_{k}^{\mathrm{zg}} & =(1-\eta)\left(G_{k}^{\mathrm{v}}\left(G_{k}^{\mathrm{v}}\right)^{\mathrm{T}}\right)+\eta R_{k} .
\end{aligned}
$$

To find the Kalman gain $K_{k}$, we resume the relations $\operatorname{tr}(M)=\operatorname{tr}\left(M^{\mathrm{T}}\right), \partial \operatorname{tr}\left(M K^{\mathrm{T}} N\right) / \partial K=M^{\mathrm{T}} N^{\mathrm{T}}$, and $\partial \operatorname{tr}\left(M K N K^{\mathrm{T}} O\right) / \partial K=N K^{\mathrm{T}} O M+N^{\mathrm{T}} K^{\mathrm{T}} M^{\mathrm{T}} O^{\mathrm{T}}$. Doing $\partial J_{k}^{\mathrm{zg}}\left(K_{k}\right) / \partial K_{k}=0$, we obtain

$$
\begin{aligned}
K_{k} & =\left(A_{k-1} P_{k-1}^{\mathrm{xx}} A_{k-1}^{\mathrm{T}}+Q_{k-1}^{\mathrm{zg}}\right) C_{k}^{\mathrm{T}} \\
& \times\left(C_{k}\left(A_{k-1} P_{k-1}^{\mathrm{xx}} A_{k-1}^{\mathrm{T}}+Q_{k-1}^{\mathrm{zg}}\right) C_{k}^{\mathrm{T}}+R_{k}^{\mathrm{zg}}\right)^{-1} .
\end{aligned}
$$

Given the Kalman gain (47), there exist many possibilities to rewrite the covariance matrix (43). By simplicity, we choose to rewrite it as (Teixeira, 2008)

$$
P_{k}^{\mathrm{gg}}=\left(\mathrm{I}_{n \times n}-K_{k} C_{k}\right)\left(A_{k-1} P_{k-1}^{\mathrm{gg}} A_{k-1}^{\mathrm{T}}+Q_{k-1}\right) .
$$

Remark 1 . Since the symmetric matrices $P_{k-1}^{\mathrm{xx}}(24)$ and $R_{k}^{\mathrm{zg}}(46)$ are positive definite, the cost function $J_{k}^{\mathrm{zg}}(14)$ is strictly convex and, then, the gain $K_{k}(47)$ is its unique global minimizer (Bernstein, 2009).

Next, we present the equivalent forms to estimate $\hat{x}_{k}^{\mathrm{h}}, \mathcal{X}_{k}$, and $X_{k}$, which characterize the hybrid state vector $x_{k}^{\mathrm{h}}$.

Algorithm 3. ZGKF: two-step form. This algorithm is summarized as

$$
\begin{array}{r}
{\left[\hat{x}_{k}^{\mathrm{h}}, G_{k}^{\mathrm{x}}, P_{k}^{\mathrm{gg}}\right]=\mathrm{ZGKF}_{-} \mathrm{TS}\left(\hat{x}_{k-1}^{\mathrm{h}}, G_{k-1}^{\mathrm{x}}, P_{k-1}^{\mathrm{gg}}, A_{k-1},\right.} \\
\left.B_{k-1}, C_{k}, G_{k-1}^{\mathrm{w}}, G_{k}^{\mathrm{v}}, Q_{k-1}, R_{k}, u_{k-1}, y_{k}, \varphi_{g}, \eta\right) .
\end{array}
$$

\section{Forecast step:}

1: Compute the a priori center

$$
\hat{x}_{k \mid k-1}^{\mathrm{h}}=A_{k-1} \hat{x}_{k-1}^{\mathrm{h}}+B_{k-1} u_{k-1} .
$$

2: Compute the matrix $\bar{G}_{k-1}^{\mathrm{x}}(23)$ to determine the a priori generator matrix

$$
G_{k \mid k-1}^{\mathrm{x}}=\left[A_{k-1} \bar{G}_{k-1}^{\mathrm{x}} G_{k-1}^{\mathrm{w}}\right] .
$$

3: Compute the matrices $P_{k-1}^{\mathrm{xx}}(24)$ and $Q_{k-1}^{\mathrm{zg}}$ (45) to obtain the a priori covariance matrices

$$
\begin{aligned}
& P_{k \mid k-1}^{\mathrm{gg}}=A_{k-1} P_{k-1}^{\mathrm{gg}} A_{k-1}^{\mathrm{T}}+Q_{k-1}, \\
& P_{k \mid k-1}^{\mathrm{zg}}=A_{k-1} P_{k-1}^{\mathrm{xx}} A_{k-1}^{\mathrm{T}}+Q_{k-1}^{\mathrm{zg}} .
\end{aligned}
$$

\section{Data-assimilation step:}

1: Compute the matrix $R_{k}^{\mathrm{zg}}(46)$ to obtain the Kalman gain $K_{k}(47)$.

2: Compute the a posteriori estimates

$$
\begin{aligned}
\hat{x}_{k}^{\mathrm{h}} & =\hat{x}_{k \mid k-1}^{\mathrm{h}}+K_{k}\left(y_{k}-C_{k} \hat{x}_{k \mid k-1}^{\mathrm{h}}\right), \\
G_{k}^{\mathrm{x}} & =\left[\left(\mathrm{I}_{n \times n}-K_{k} C_{k}\right) G_{k \mid k-1}^{\mathrm{x}}-K_{k} G_{k}^{\mathrm{v}}\right], \\
P_{k}^{\mathrm{gg}} & =\left(\mathrm{I}_{n \times n}-K_{k} C_{k}\right) P_{k \mid k-1}^{\mathrm{gg}} .
\end{aligned}
$$

Algorithm 4. ZGKF: one-step form. This algorithm is summarized as

$$
\begin{array}{r}
{\left[\hat{x}_{k}^{\mathrm{h}}, G_{k}^{\mathrm{x}}, P_{k}^{\mathrm{gg}}\right]=\text { ZGKF_OS }\left(\hat{x}_{k-1}^{\mathrm{h}}, G_{k-1}^{\mathrm{x}}, P_{k-1}^{\mathrm{gg}}, A_{k-1},\right.} \\
\left.B_{k-1}, C_{k}, G_{k-1}^{\mathrm{w}}, G_{k}^{\mathrm{v}}, Q_{k-1}, R_{k}, u_{k-1}, y_{k}, \varphi_{g}, \eta\right) .
\end{array}
$$

1: Compute the matrices $\bar{G}_{k-1}^{\mathrm{x}}(23), P_{k-1}^{\mathrm{xx}}(24), Q_{k-1}^{\mathrm{zg}}(45)$, and $R_{k}^{\mathrm{zg}}(46)$ to obtain the Kalman gain $K_{k}(47)$.

2: Compute the a posteriori estimates

$$
\begin{aligned}
\hat{x}_{k}^{\mathrm{h}} & =\left(\mathrm{I}_{n \times n}-K_{k} C_{k}\right)\left(A_{k-1} \hat{x}_{k-1}^{\mathrm{h}}+B_{k-1} u_{k-1}\right)+K_{k} y_{k}, \\
G_{k}^{\mathrm{x}} & =\left[\left(\mathrm{I}_{n \times n}-K_{k} C_{k}\right)\left[A_{k-1} \bar{G}_{k-1}^{\mathrm{x}} G_{k-1}^{\mathrm{w}}\right]-K_{k} G_{k}^{\mathrm{v}}\right],
\end{aligned}
$$
and (48).

\section{NUMERICAL EXAMPLE}

\subsection{Process Description}

Consider the discrete-time linear system (Alamo et al., 2005)

$$
\begin{aligned}
& x_{k}=\left[\begin{array}{cc}
0 & -0.5 \\
1 & 1
\end{array}\right] x_{k-1}+0.02\left[\begin{array}{c}
-6 \\
1
\end{array}\right] w_{k-1}, \\
& y_{k}=\left[\begin{array}{ll}
-2 & 1
\end{array}\right] x_{k}+0.2 v_{k},
\end{aligned}
$$

where $w_{k-1}$ and $v_{k}$ are realizations taken from the GRV $W_{k-1} \sim \mathcal{N}(0,1)$ and $\mathrm{ZV} \mathcal{V}_{k}=\{0,1\}$, respectively. In this case, the values $v_{k}$ are yielded by a uniform variable defined into the unitary interval $[-1,1]$. In turn, the initial state is set as $x_{0}=0_{2 \times 1}$.

To estimate the states, we apply the ZGKP and ZGKF algorithms. The estimators are set as $\eta=0.5, \alpha=0.0027$, $\varphi_{g}=14, \hat{x}_{0}^{\mathrm{h}}=\left[\begin{array}{ll}0.5 & 0.5\end{array}\right]^{\mathrm{T}}, G_{0}^{\mathrm{x}}=0.60 \times \mathrm{I}_{2 \times 2}, P_{0}^{\mathrm{gg}}=0.040 \times$ $\mathrm{I}_{2 \times 2}, Q_{k-1}=1$, and $G_{k}^{\mathrm{v}}=1$. The reduction order $\varphi_{g}$ is chosen based on some offline simulations such that $\varphi_{g}>14$ leads to similar results. The parameter $\eta=0.5$ is set according to the same quantity of existing uncertainties in the system. For the parameter $\alpha=0.0027$, we have the usual $99.73 \%$ confidence level. Given $\alpha$, we set $\hat{x}_{0}^{\mathrm{h}}, G_{0}^{\mathrm{x}}$, and $P_{0}^{\text {gg }}$ such that the confidence box (12) includes the initial state $x_{0}$.

\subsection{State Estimation}

The results of the state estimation are presented in Figure 2. Graphs (a) and (b) show the a posteriori centers $\hat{x}_{k}^{\mathrm{h}}$ and confidence boxes $\mathcal{I}^{\alpha}$ computed by (12). Observe that the ZGKF provides: (i) punctual estimates closer to the true states, and (ii) confidence boxes smaller than the ZGKP. It occurs because the filter is able to incorporate larger information during the data-assimilation step, then, it is able to correct the predictions more efficiently than the predictor. Graph (c) shows the Frobenius norm, computed by (9), of the confidence boxes $\mathcal{I}^{\alpha}$ related to both ZGKP and ZGKF. The difference between each norm, at the same time step, illustrates the overall uncertainty reduction reached by the filter in comparison to the predictor. 


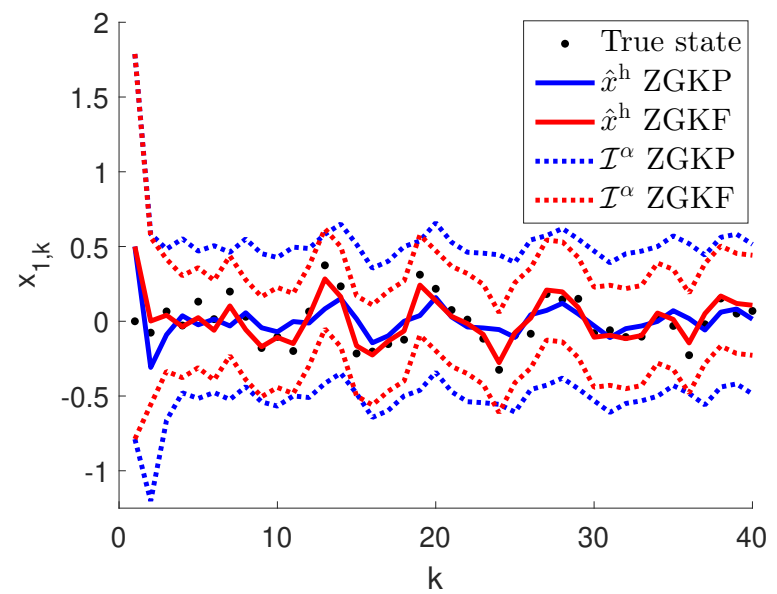

(a)

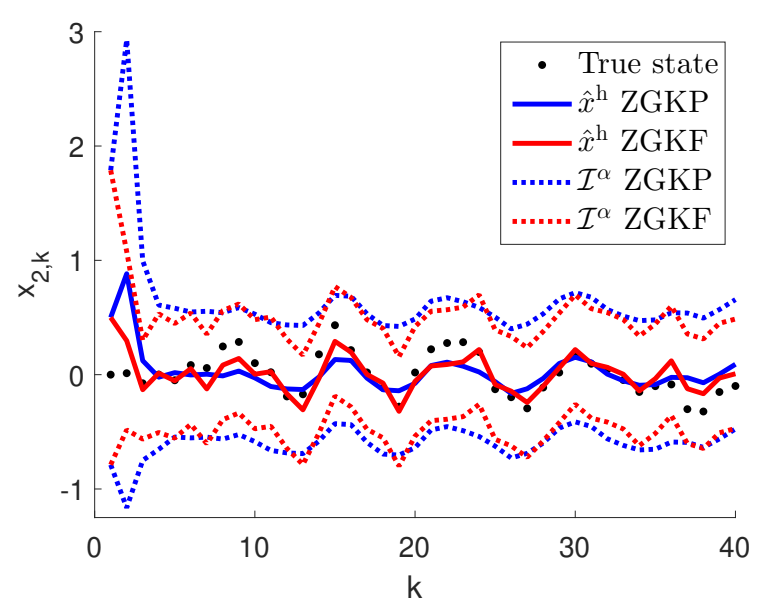

(b)

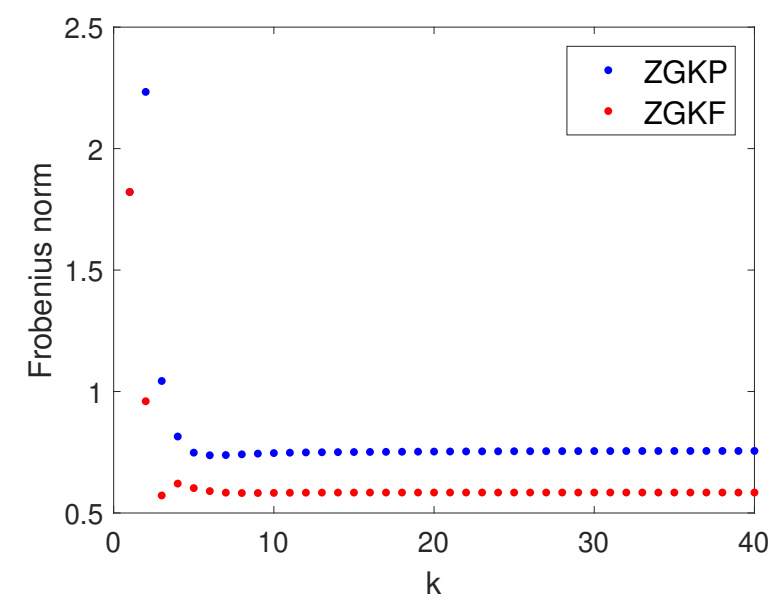

(c)

Figure 2. State estimation for a two-state linear system. Graphs (a) and (b) illustrate the confidence intervals $(:)$ and centers $(-)$ generated from the ZGKP (blue color) and ZGKF (red color) algorithms to represent the true states (.). Graph (c) illustrates the Frobenius norm of the confidence boxes computed over time.

\section{CONCLUSION}

This paper revisited the original zonotopic and Gaussian Kalman estimator, here called ZGKP, in two equivalent forms, namely one-step and two-step. The former computes all estimates at once, while the latter computes the same estimates making explicit the process and measurement model contributions. This is important to differ some state estimator classes such as predictors and filters, since the difference is the considered measurement sequence. On one hand, predictors as the ZGKP use only past measurements. On the other hand, filters as the ZGKF use both past and current measurements. Moreover, this paper proposed the filter version to the ZGKP, here called ZGKF, in the one-step and two-step equivalent forms. A numerical example is proposed to illustrate the advantages obtained by using the filter instead of the predictor.

\section{REFERENCES}

Alamo, T., Bravo, J.M., and Camacho, E.F. (2005). Guaranteed state estimation by Zonotopes. Automatica, 41(6), 1035-1043.

Bernstein, D.S. (2009). Matrix Mathematics: Theory, Facts, and Formulas Ed. 2. Princeton University Press.

Combastel, C. (2003). A state bounding observer based on zonotopes. In European Control Conference (ECC), 2003, 2589-2594.

Combastel, C. (2015b). Merging Kalman filtering and zonotopic state bounding for robust fault detection under noisy environment. IFAC-PapersOnLine, 48(21), 289-295.

Combastel, C. (2015a). Zonotopes and Kalman observers: Gain optimality under distinct uncertainty paradigms and robust convergence. Automatica, 55, 265-273.

de Paula, A.A., Raffo, G.V., and Teixeira, B.O.S. (2018). State estimation based on stochastic and zonotopic approaches: part I - linear systems. In XXII Congresso Brasileiro de Automática, 2018, João Pessoa, 1-8. Anais do XXII Congresso Brasileiro de Automática.

Jaulin, L. and Kieffer, M. (2001). Applied Interval Analysis. Springer.

Kalman, R.E. (1960). A new approach to linear filtering and prediction problems. Journal of basic Engineering, $82(1), 35-45$.

Kim, I.S., Teixeira, B.O., and Bernstein, D.S. (2008). Ensemble-on-demand kalman filter for large-scale systems with time-sparse measurements. In 200847 th IEEE Conference on Decision and Control, 3199-3204.

Kim, I.S., Teixeira, B.O., Chandrasekar, J., and Bernstein, D.S. (2009). Reduced-order state estimation for linear time-varying systems. Asian Journal of Control, 11(6), 595-609.

Kühn, W. (1998). Rigorously computed orbits of dynamical systems without the wrapping effect. Computing, 61(1), 47-67.

Le, V.T.H., Alamo, T., Camacho, E.F., Stoica, C., and Dumur, D. (2012). Zonotopic set-membership estimation for interval dynamic systems. In 2012 American Control Conference (ACC), 6787-6792.

Le, V.T.H., Stoica, C., Alamo, T., Camacho, E.F., and Dumur, D. (2013). Zonotopes: From guaranteed stateestimation to control. John Wiley \& Sons.

Moore, R.E., Kearfott, R.B., and Cloud, M.J. (2009). Introduction to interval analysis. SIAM.

Teixeira, B.O.S. (2008). Kalman filters [Ask the Experts]. IEEE Control Systems Magazine, 28(2), 16-18. 